\title{
The variable finesse locking technique
}

\author{
F Acernese ${ }^{1}$, P Amico $^{2}$, M Al-Shourbagy ${ }^{3}$, S Aoudia $^{4}$, S Avino $^{1}$,
} D Babusci $^{5}$, G Ballardin ${ }^{6}, \mathbf{R}$ Barillé $^{6}, \mathbf{F}$ Barone $^{1}$, L Barsotti $^{3}$, M Barsuglia $^{7}$, F Beauville $^{8}$, M A Bizouard ${ }^{7}, \mathbf{C}$ Boccara $^{9}$, F Bondu $^{4}$,

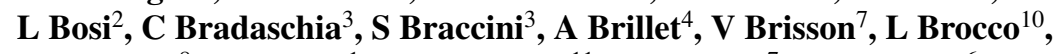
D Buskulic ${ }^{8}$, E Calloni ${ }^{1}$, E Campagna ${ }^{11}$, F Cavalier $^{7}$, R Cavalieri $^{6}$, G Cella ${ }^{3}$, E Chassande-Mottin ${ }^{4}$, C Corda $^{3}$, A-C Clapson ${ }^{7}$, F Cleva $^{4}$, J-P Coulon ${ }^{4}$, E Cuoco ${ }^{6}$, V Dattilo ${ }^{6}$, M Davier ${ }^{7}$, R De Rosa ${ }^{1}$, L Di Fiore ${ }^{1}$, A Di Virgilio ${ }^{3}$, B Dujardin ${ }^{4}$, A Eleuteri ${ }^{1}$, D Enard ${ }^{6}$, I Ferrante ${ }^{3}$, F Fidecaro $^{3}$, I Fiori ${ }^{3}$, R Flaminio ${ }^{6,8}$, J-D Fournier ${ }^{4}$, S Frasca ${ }^{10}$, F Frasconi $^{3,6}$, A Freise ${ }^{6}$, L Gammaitoni ${ }^{2}$, G Gennai $^{3}$, A Giazotto $^{3}$, G Giordano $^{5}$, L Giordano ${ }^{1}$, R Gouaty ${ }^{8}$, D Grosjean ${ }^{8}$, G Guidi ${ }^{11}$, S Hebri ${ }^{6}$, H Heitmann $^{4}$, P Hello ${ }^{7}$ L Holloway ${ }^{6}$, S Kreckelbergh ${ }^{7}$, P La Penna ${ }^{6}$, V Loriette $^{9}$, M Loupias ${ }^{6}$, G Losurdo $^{11}$, J-M Mackowski ${ }^{12}$, E Majorana ${ }^{10}$, C N Man ${ }^{4}, \mathbf{M}$ Mantovani ${ }^{3}$, F Marchesoni ${ }^{2}$, F Marion ${ }^{8}, \mathbf{J ~ M a r q u e ~}^{6}$, F Marteli ${ }^{11}$, A Masserot ${ }^{8}$, M Mazzoni $^{11}$, L Milano $^{1}$, C Moins $^{6}$, J Moreau ${ }^{9}$, N Morgado ${ }^{12}$, B Mours ${ }^{8}$, A Pai ${ }^{10}$, C Palomba ${ }^{10}$, F Paoletti ${ }^{3,6}$, S Pardi ${ }^{1}$, A Pasqualetti ${ }^{6}, \mathbf{R}$ Passaquieti ${ }^{3}$, D Passuello ${ }^{3}$, B Perniola $^{11}$, F Piergiovanni ${ }^{11}$, L Pinard $^{12}$, R Poggiani $^{3}$, M Punturo $^{2}$, P Puppo $^{10}$, K Qipiani ${ }^{1}$, P Rapagnani ${ }^{10}$, V Reita ${ }^{9}$, A Remillieux ${ }^{12}$, F Ricci $^{10}$, I Ricciardi ${ }^{1}$, P Ruggi ${ }^{6}$, G Russo ${ }^{1}$, S Solimeno ${ }^{1}$, A Spallicii ${ }^{4}$, R Stanga ${ }^{11}$, R Taddei ${ }^{6}$, D Tombolato ${ }^{8}$, M Tonelli $^{3}$, A Toncelli ${ }^{3}$, E Tournefier ${ }^{8}$,

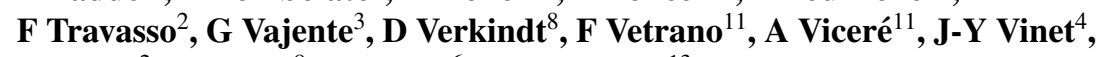

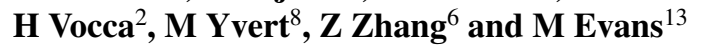

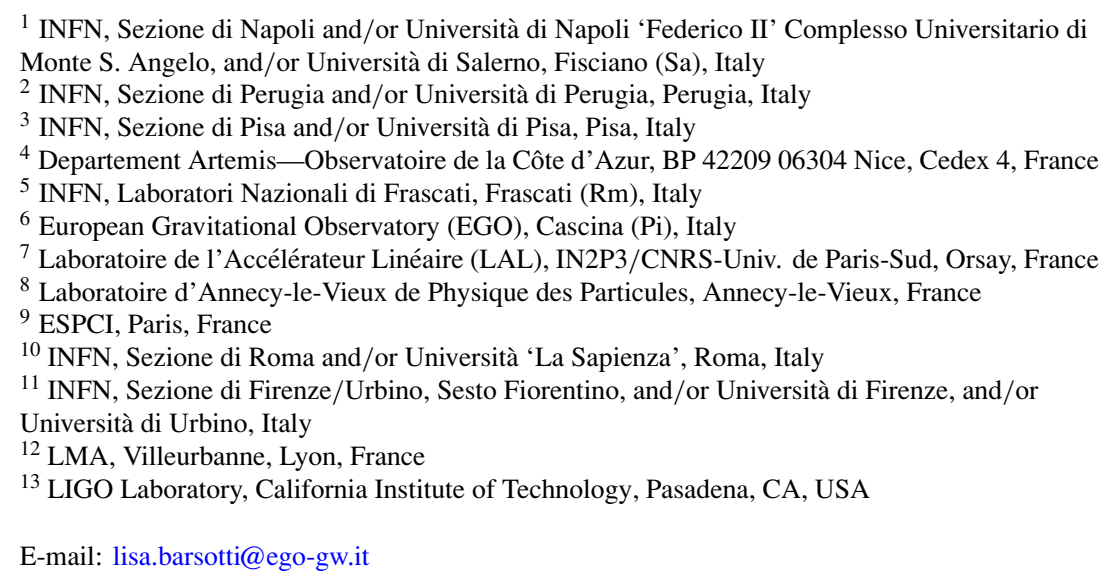

Received 29 August 2005

Published 24 March 2006

Online at stacks.iop.org/CQG/23/S85 


\begin{abstract}
Virgo is a power recycled Michelson interferometer, with $3 \mathrm{~km}$ long FabryPerot cavities in the arms. The locking of the interferometer has been obtained with an original lock acquisition technique. The main idea is to lock the instrument away from its working point. Lock is obtained by misaligning the power recycling mirror and detuning the Michelson from the dark fringe. In this way, a good fraction of light escapes through the antisymmetric port and the power build-up inside the recycling cavity is extremely low. The benefit is that all the degrees of freedom are controlled when they are almost decoupled, and the linewidth of the recycling cavity is large. The interferometer is then adiabatically brought on to the dark fringe. This technique is referred to as variable finesse, since the recycling cavity is considered as a variable finesse Fabry-Perot. This technique has been widely tested and allows us to reach the dark fringe in few minutes, in an essentially deterministic way.
\end{abstract}

PACS numbers: $04.80 . \mathrm{Nn}, 95.55 . \mathrm{Ym}$

(Some figures in this article are in colour only in the electronic version)

\title{
1. The Virgo longitudinal control system
}

The nominal sensitivity of a power recycled Michelson interferometer detector with suspended optics such as Virgo is achieved by selecting an appropriate working point, with laser light resonant in the optical cavities, and the output port tuned on the dark fringe. These conditions translate into fixed relationships between the laser light wavelength and four independent lengths of the interferometer (see figure 1):

- the length of the recycling cavity (PRCL), $l_{\text {rec }}+\frac{l_{N}+l_{W}}{2}$;

- the differential length of the short Michelson arms (MICH), $l_{N}-l_{W}$;

- the common (CARM) and the differential (DARM) length of the two long arms, $L_{N}+L_{W}$ and $L_{N}-L_{W}$.

The Virgo suspension system, the so-called superattenuator (SA) [1], provides very good seismic isolation in the frequency band used for detection, but below a few hertz some seismic noise is transferred to the mirrors or even amplified by the SA mechanical resonances. While the expected sensitivity is of the order of $10^{-18} \mathrm{~m} \mathrm{~Hz}^{-1 / 2}$ at the rate of $10 \mathrm{~Hz}$, the allowed deviation from the working point, or locking accuracy, is $10^{-12} \mathrm{~m} \mathrm{rms}$. An active feedback control system is therefore needed to keep the interferometer locked on the required interference conditions.

Relative displacement of the mirrors is detected using a carrier beam phase modulated at $f=6 \mathrm{MHz}$. The carrier beats with the sidebands, producing light modulated in intensity with frequency $f, 2 f, \ldots$ Using a standard Pound-Drever-Hall scheme $[2,3]$ all the lengths involved can be reconstructed by mixing the signals produced by the photodiodes, which are placed at different output ports of the interferometer. These error signals are digitized and sent to the Virgo global control system (Global Control [4]), which computes the corrections to be applied to the mirrors by coil magnet actuators. A local control system, referred to as the ground, is active in the bottom part of each SA in order to keep the longitudinal displacement of the mirrors below $1 \mu \mathrm{m}$ rms. In this way, the longitudinal lock of the interferometer can be acquired using a limited actuation force, thus preventing noise reinjection in the detection band. 


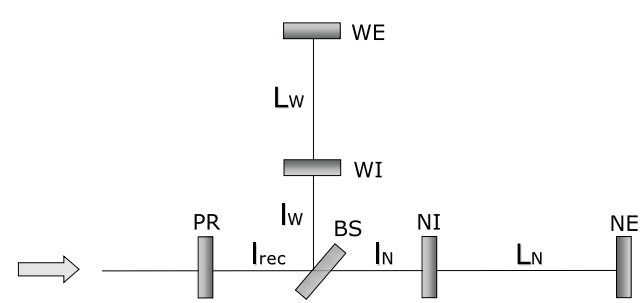

Figure 1. Optical scheme of Virgo.

\section{Lock acquisition of the recycled interferometer}

The process by which an interferometer in an initially uncontrolled condition is brought to and kept at its working point by closing the feedback loops is called lock acquisition.

Detectors with an optical set-up similar to Virgo, such as LIGO and TAMA, apply a lock acquisition technique based on a statistical approach, where the control loops are engaged every time the interferometer passes through the relative resonance conditions. The four independent lengths of the interferometer are then sequentially locked at their operating points, dynamically changing the optical sensing matrix in order to compensate the variation of the fields in the course of lock acquisition [6]. The locked state is reached in a sequence of three steps ${ }^{14}$ starting from the uncontrolled condition, where only the final one is stable.

\subsection{The variable finesse locking technique}

An alternative lock acquisition technique has been developed in Virgo: it differs from the multisteps scheme mainly because all four longitudinal degrees of freedom of the interferometer are locked simultaneously.

The main idea is that the interferometer is locked away from the working point of the dark fringe. A good fraction of light escapes through the antisymmetric port and the power build-up inside the recycling cavity is extremely low. In this way, all the lengths of the interferometer are controlled when they are almost decoupled and the linewidth of the recycling cavity is large, making the control design easier. From this stable state, the interferometer is then adiabatically brought on to the dark fringe. This technique is referred to as variable finesse, because the finesse of the recycling cavity changes during the lock acquisition path.

\subsection{The variable finesse locking procedure}

The locking procedure starts with the PR mirror slightly misaligned by some microradians, in order to further decrease the power stored inside the recycling cavity. The simple Michelson is kept at mid-fringe (50\% reflected, 50\% transmitted), adding an offset in the dark port dc signal and applying the correction to the BS mirror. Since all the degrees of freedom are almost decoupled in this state, the two arms can be independently locked using the end photodiodes. The small quantity of light reflected by the interferometer is used to control the recycling cavity power length, by the reflected $3 f$-demodulated signal, as comprehensively tested in the TAMA control scheme [7]. This stable configuration can usually be reached

${ }^{14}$ In the first step, the sidebands enter in resonance in the recycling cavity (anti-resonant in the arms), the carrier is on the anti-resonance for the recycling cavity and for the arms; in the second step, the carrier enters in resonance in one arm, on the bright fringe in the recycling cavity, where the sidebands remain in the same condition; in the last step, the carrier goes in resonance in both arms and in the recycling cavity [5]. 


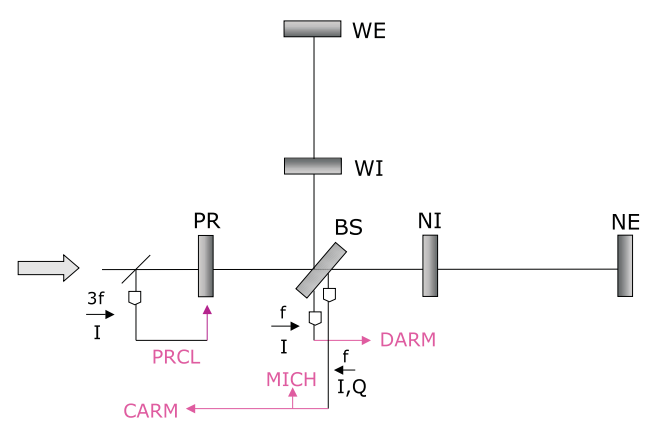

Figure 2. Final locking control scheme: PRCL is controlled using the reflected $3 f$-demodulated signal, MICH by one phase of the demodulated signal extracted from the second face of BS, the other phase is used to control CARM. DARM is controlled by the dark port demodulated signal.

in a few seconds, with a pre-alignment of all the mirrors within $0.5 \mu \mathrm{rad}$, preventing mirror excitations. From this starting condition the PR is realigned, while always maintaining the Michelson at mid-fringe, giving a very low recycling gain.

In order to increase the recycling gain, the Michelson has to be brought on to the dark fringe: this is done adiabatically, decreasing the offset in the Michelson error signal. At the same time, the control scheme evolves to take into account the increasing coupling between the different degrees of freedom. The end photodiodes, in fact, can only be used to independently control the cavities when the interferometer is far from the dark fringe. When approaching the dark fringe they begin to couple strongly and a common and differential control has to be activated to maintain the interferometer locked.

Then a frequency stabilization servo is engaged, controlling CARM with a bandwidth of a few kilohertz: consequently, the signal generated by this degree of freedom on all the photodiodes is significantly reduced. DARM is kept in a locked state by one of the end photodiode signals. The final step consists of switching from the dc to a demodulated signal to control the Michelson length. Eventually, the offset in the Michelson error signal is removed; the interferometer goes on to the dark fringe and the recycling cavity gain increases up to the maximum value. The last step of the lock acquisition procedure (see figure 2) consists of moving the DARM control from the end photodiode signal to the dark port signal, which has a better signal-to-noise ratio.

The PRCL length remains stably controlled by the reflected $3 f$-demodulated signal during the entire lock acquisition sequence. This is in fact the main advantage in using the $3 f$-demodulation scheme with respect to the standard $f$ scheme for the PRCL control: the stability of amplitude and sign of the PRCL reconstructed length even during lock acquisition, which allows us to keep this degree of freedom locked without changing the control scheme [8].

\section{Results and conclusions}

The lock acquisition sequence usually takes less than $2 \mathrm{~min}$, as shown in figure 3 ; thanks to a fully automatic locking procedure.

During the commissioning run C6 [9], Virgo ran for two weeks in recycled configuration. The interferometer has been systematically locked applying the variable finesse technique, in a deterministic and repeatable way. The duty cycle over the two weeks has been of $86 \%$, with a longest lock lasting 40 hours (see figure 4). 


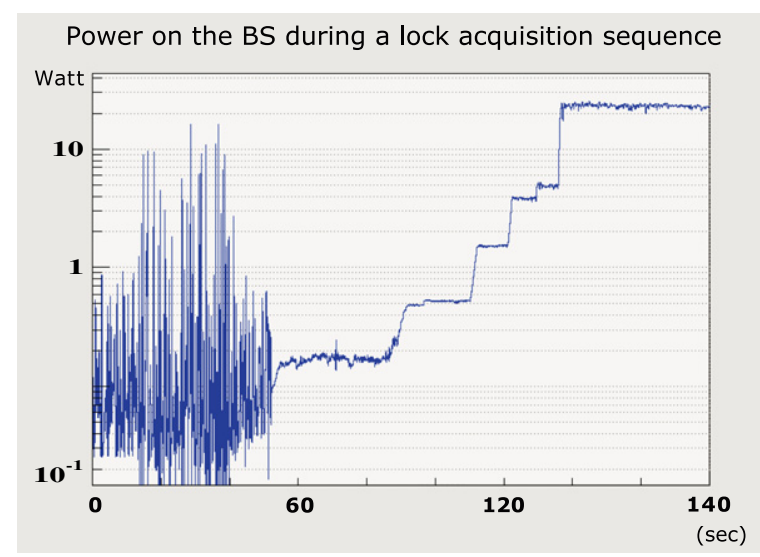

Figure 3. Lock acquisition sequence, looking at the power impinging on the BS: the interferometer is brought from the uncontrolled to the fully controlled state in less than $2 \mathrm{~min}$.

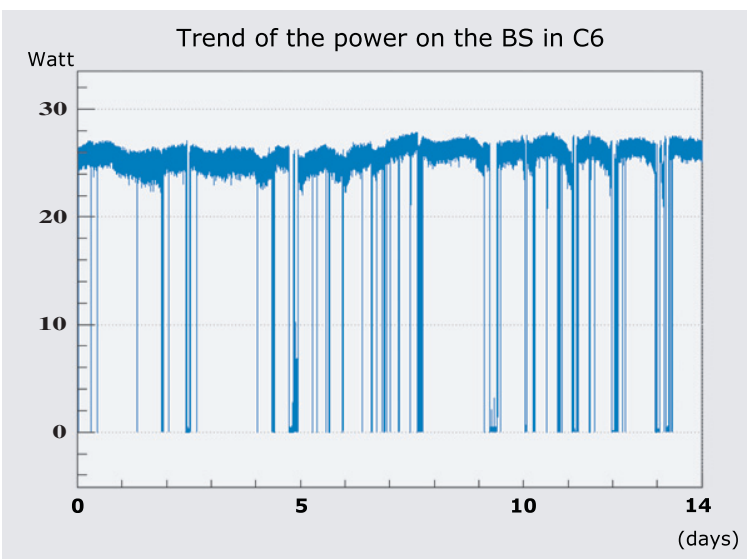

Figure 4. Trend of the power impinging on the BS during 14 days of data taking (C6): the duty cycle is around $86 \%$.

\section{References}

[1] Ballardin G et al 2001 Measurement of the VIRGO superattenuator performance for seismic noise suppression Rev. Sci. Instrum. 72 3643-52

[2] Pound R V 1946 Electronic frequency stabilization of microwave oscillators Rev. Sci. Instrum. 17 490-505

[3] Drever R W P et al 1983 Laser phase and frequency stabilization using an optical resonator Appl. Phys. B 31 97-105

[4] Cavalier F 2001 Le controle global de Virgo These d'Habilitation a diriger des Recherches Universitè de Paris Sud, LAL 01-69

[5] Evans M 2001 Lock acquisition in resonant optical interferometers PhD Thesis CALTECH

[6] Evans M et al 2002 Lock acquisition of a gravitational-wave interferometer Opt. Lett. 27 598-600

[7] Arai K (TAMA Collaboration) 2002 Sensing and controls for power-recycling of TAMA300 Class. Quantum Grav. $191843-8$

[8] Arai K 2001 Robust exctraction of control signals for power-recycled interferometric gravitational-wave detectors PhD Thesis University of Tokyo

[9] Acernese F et al 2006 Class. Quantum Grav. 23 S63 